TRANSACTIONS OF THE

AMERICAN MATHEMATICAL SOCIETY

Volume 363, Number 3, March 2011, Pages 1463-1485

S 0002-9947(2010)05176-1

Article electronically published on October 13, 2010

\title{
GENERALIZED LOG SINE INTEGRALS AND THE MORDELL-TORNHEIM ZETA VALUES
}

\author{
KAZUHIRO ONODERA
}

\begin{abstract}
We introduce certain integrals of a product of the Bernoulli polynomials and logarithms of Milnor's multiple sine functions. It is shown that all the integrals are expressed by the Mordell-Tornheim zeta values at positive integers and that the converse is also true. Moreover, we apply the theory of the integral to obtain various new results for the Mordell-Tornheim zeta values.
\end{abstract}

\section{INTRODUCTION}

Integrals involving the logarithm of the trigonometric function were first studied by Euler [6, 7]. He showed that

$$
\int_{0}^{\frac{\pi}{2}} \log (\sin x) d x=-\frac{\pi}{2} \log 2
$$

and

$$
\int_{0}^{\frac{\pi}{2}} x \log (\sin x) d x=\frac{7}{16} \zeta(3)-\frac{\pi^{2}}{8} \log 2 .
$$

After his discovery, many people studied their generalizations and analogues: (a) integrals on a different interval, (b) indefinite integrals, (c) integrals involving products of a polynomial of higher degree and logarithms of trigonometric functions, (d) $q$-analogues, and (e) integrals of a logarithm of generalized multiple sine function. These works can be, for instance, found in [19] for (a)-(c), in [14, 17] for (d), and in 13, 15, 16, 18, for (e). It is attractive to build a theory that enables us to handle some of them together. In particular, our present concern is the construction of a theory involving the works for (c) and (e). In this paper, as a first step of this research, we concentrate on the case of Milnor's multiple sine function $\mathbf{S}_{r}(x)$ (which, while inspired by [24], is actually defined in [16]) whose logarithm is equal to a certain constant multiple of the Clausen function $\mathrm{Cl}_{r}(2 \pi x)$ in the interval $(0,1)$. Concretely, we study the integral

$$
I_{p, q}(\boldsymbol{a} ; \boldsymbol{b}):=\int_{0}^{1} \prod_{i=1}^{p} B_{a_{i}}(x) \prod_{j=1}^{q} b_{j} \log \mathbf{S}_{b_{j}}(x) d x
$$

Received by the editors December 6, 2008 and, in revised form, June 1, 2009.

2000 Mathematics Subject Classification. Primary 11M06, 11M35, 33E20.

Key words and phrases. Log sine integral, multiple zeta value, multiple sine function, Clausen function, Mordell-Tornheim zeta value.

(C)2010 American Mathematical Society Reverts to public domain 28 years from publication 
for non-negative integers $p, q$ and $\boldsymbol{a}=\left(a_{1}, \ldots, a_{p}\right) \in\left(\mathbb{Z}_{\geq 0}\right)^{p}, \boldsymbol{b}=\left(b_{1}, \ldots, b_{q}\right) \in$ $\left(\mathbb{Z}_{\geq 1}\right)^{q}$, where $B_{a}(x)$ is the Bernoulli polynomial. The integral $I_{p, q}(\boldsymbol{a} ; \boldsymbol{b})$ is sometimes written as $I_{p, q}\left(a_{1}, \ldots, a_{p} ; b_{1}, \ldots, b_{q}\right)$. When $p=0$ or $q=0$, it is understood that the empty product is equal to 1 , and $I_{p, q}(\boldsymbol{a} ; \boldsymbol{b})$ is denoted by $I_{0, q}(\boldsymbol{b})=$ $I_{0, q}\left(b_{1}, \ldots, b_{q}\right)$ or $I_{p, 0}(\boldsymbol{a})=I_{p, 0}\left(a_{1}, \ldots, a_{p}\right)$, respectively.

The purpose of this paper is to show basic formulas of the integral $I_{p, q}$ and obtain its explicit expression such as Euler's work. It does not appear that these integrals can in general be described in terms of ordinary zeta values, but rather in terms of the Mordell-Tornheim zeta values

$$
\zeta_{M T, r}\left(s_{1}, \ldots, s_{r} ; s_{r+1}\right)=\sum_{n_{1}, \ldots, n_{r}=1}^{\infty} \frac{1}{n_{1}^{s_{1}} \cdots n_{r}^{s_{r}}\left(n_{1}+\cdots+n_{r}\right)^{s_{r+1}}}
$$

for a positive integer $r$, which was defined as a function of complex variables by Matsumoto 21, 22. The series on the right hand side converges absolutely for $s_{1}, \ldots, s_{r+1} \in \mathbb{C}$ satisfying the fact that $\operatorname{Re}\left(s_{i_{1}}+\cdots+s_{i_{k}}+s_{r+1}\right)>k$ if $k=1, \ldots, r$ and $1 \leq i_{1}<\cdots<i_{k} \leq r$. The function $\zeta_{M T, r}\left(s_{1}, \ldots, s_{r} ; s_{r+1}\right)$ is meromorphically continued to the whole space $\mathbb{C}^{r+1}$, and its possible singularities are explicitly determined (see [22, Theorem 1]). The special values of the Mordell-Tornheim zeta function at positive integers play the important role in this paper. Actually, after an explanation of the basic properties of the integral $I_{p, q}$ in $\S 2$, we show in the first half of $\S 3$ that the Mordell-Tornheim zeta value at positive integers is concretely expressed as a linear combination of the integrals and that the converse is also true. A simple example is as follows: if $a, b, c$ are positive integers and $a+b+c$ is even, then

$$
\zeta_{M T, 2}(a, b ; c)=\frac{(-1)^{\frac{a+b-c}{2}}(2 \pi)^{a+b+c-2}}{2 a ! b ! c !}\left(I_{1,2}(c ; a, b)-\pi^{2} I_{3,0}(a, b, c)\right),
$$

and the formula for $I_{1,2}(c ; a, b)$ follows from Carlitz's calculation of $I_{3,0}(a, b, c)$ (see [3. (4)] or (4.22) ). These results suggest that the theory of the integral $I_{p, q}$ and that of the Mordell-Tornheim zeta values are applicable to each other. In this paper, we apply properties of the integral to prove several results of the Mordell-Tornheim zeta values. In the second half of $\S 3$, we realize an explicit formulation for the parity result of Tsumura [34, Theorem 1.1] and show another value-relation among the Mordell-Tornheim zeta values. In $\S 4$, we investigate some cases in which $I_{p, q}$ is described by the Riemann zeta values. We first show each generating function of $I_{1, q}(a ; 1, \ldots, 1)$ and $\zeta_{M T, r}(1, \ldots, 1 ; a)$ and obtain their explicit expressions. Next, some of the integrals $I_{p, q}$ with $q \leq 3$ are studied concretely. As a result, we attain many expressions for the Mordell-Tornheim zeta values by the Riemann zeta values, which contain most of the known results and some new relations for $\zeta_{M T, r}(r \geq 3)$. For example, we obtain

$$
\begin{aligned}
& \zeta_{M T, 3}(2,1,1 ; 1)=2 \zeta(2) \zeta(3)-\zeta(5), \\
& \zeta_{M T, 3}(4,1,1 ; 1)=-2^{-1} \zeta(3) \zeta(4)+4 \zeta(2) \zeta(5)-4 \zeta(7) .
\end{aligned}
$$

Remark on Notation. Our special notation is listed here: let $m$ be a positive integer and let $\boldsymbol{x}=\left(x_{1}, \ldots, x_{m}\right)$ be a row vector.

(a) $N_{m}:=\{1, \ldots, m\}$.

(b) $|\boldsymbol{x}|:=x_{1}+\cdots+x_{m}$

(c) $\boldsymbol{x}_{K}:=\left(x_{i_{1}}, \ldots, x_{i_{k}}\right)$ for a set $K=\left\{i_{1}, \ldots, i_{k}\right\} \subset N_{m}$ with $1 \leq i_{1}<\cdots<$ $i_{k} \leq m$. 
(d) The multinomial coefficient is extended as follows:

$$
\left(\begin{array}{c}
N \\
n_{1}, \ldots, n_{k}
\end{array}\right):= \begin{cases}\frac{N !}{n_{1} ! \cdots n_{k} !} & \text { if } n_{1}, \ldots, n_{k} \in \mathbb{Z}_{\geq 0} \text { and } N=\sum_{j=1}^{k} n_{j} \\
0 & \text { otherwise. }\end{cases}
$$

(e) Each function can take any parameter of various forms that are suitable for the number of its variables. For example, it is understood that $I_{p, q}\left(a_{1}, \boldsymbol{a}^{\prime}, \boldsymbol{a}^{\prime \prime} ; \boldsymbol{b}^{\prime}\right.$, $\left.b_{3}, \ldots, b_{q}\right)$ with $\boldsymbol{a}^{\prime}=\left(a_{2}, a_{3}\right), \boldsymbol{a}^{\prime}=\left(a_{4}, \ldots, a_{p}\right)$ and $\boldsymbol{b}^{\prime}=\left(b_{1}, b_{2}\right)$ equals $I_{p, q}(\boldsymbol{a} ; \boldsymbol{b})$ with $\boldsymbol{a}=\left(a_{1}, \ldots, a_{p}\right)$ and $\boldsymbol{b}=\left(b_{1}, \ldots, b_{q}\right)$.

\section{BASIC FORMUlAS FOR GENERALIZED LOG SINE INTEGRALS}

We first recall basic properties of Milnor's multiple sine function (refer to [16]). Let $r$ be a positive integer. Then we define Milnor's multiple sine function by

$$
\mathbf{S}_{r}(x)=\boldsymbol{\Gamma}_{r}(x)^{-1} \boldsymbol{\Gamma}_{r}(1-x)^{(-1)^{r}}
$$

for $x \in(0,1)$, where

$$
\boldsymbol{\Gamma}_{r}(x)=\exp \left(\left.\frac{\partial}{\partial s} \zeta(s, x)\right|_{s=1-r}\right)
$$

and $\zeta(s, x)$ is the Hurwitz zeta function. It immediately follows that $\mathbf{S}_{r}(1-x)=$ $\mathbf{S}_{r}(x)^{(-1)^{r-1}}$. The functional equation of the Hurwitz zeta function yields that the logarithm of Milnor's multiple sine function has the following Fourier expansion in the interval $(0,1)$. For an odd integer $r \geq 1$,

$$
\log \mathbf{S}_{r}(x)=(-1)^{\frac{r+1}{2}} \frac{(r-1) !}{(2 \pi)^{r-1}} \sum_{n=1}^{\infty} \frac{\cos (2 \pi n x)}{n^{r}},
$$

and for an even integer $r \geq 2$,

$$
\log \mathbf{S}_{r}(x)=(-1)^{\frac{r}{2}} \frac{(r-1) !}{(2 \pi)^{r-1}} \sum_{n=1}^{\infty} \frac{\sin (2 \pi n x)}{n^{r}}
$$

Thus, we see that

$$
\left.\log \mathbf{S}_{r}(x)=(-1)^{\left\lfloor\frac{r+1}{2}\right.}\right\rfloor \frac{(r-1) !}{(2 \pi)^{r-1}} \mathrm{Cl}_{r}(2 \pi x)
$$

for $x \in(0,1)$, where $\mathrm{Cl}_{r}(x)$ is the Clausen function. Moreover, we can show that $\frac{d}{d x} \log \mathbf{S}_{r}(x)=(r-1) \log \mathbf{S}_{r-1}(x)$ for $r \geq 2$ and that there exists the limit of $\log \mathbf{S}_{r}(x)$ for $r \geq 2$ as $x \downarrow 0$. The limit is denoted by $\log \mathbf{S}_{r}(0)$, and so

$$
\log \mathbf{S}_{r}(0)= \begin{cases}(-1)^{\frac{r+1}{2}} \frac{(r-1) !}{(2 \pi)^{r-1}} \zeta(r) & \text { if } r \text { is an odd integer } \geq 3 \\ 0 & \text { if } r \text { is an even integer } \geq 2 .\end{cases}
$$

We now apply the above properties of Milnor's multiple sine function to show basic formulas for the integral $I_{p, q}$.

Proposition 2.1. Let $p$ and $q$ be non-negative integers. Let $\boldsymbol{a}=\left(a_{1}, \ldots, a_{p}\right) \in$ $\left(\mathbb{Z}_{\geq 0}\right)^{p}$ and $\boldsymbol{b}=\left(b_{1}, \ldots, b_{q}\right) \in\left(\mathbb{Z}_{\geq 1}\right)^{q}$. If $|\boldsymbol{a}|+|\boldsymbol{b}|+q$ is odd, then $I_{p, q}(\boldsymbol{a} ; \boldsymbol{b})=0$. 
Proof. Since $B_{a}(1-x)=(-1)^{a} B_{a}(x)(a \geq 0)$ and $\log \mathbf{S}_{b}(1-x)=(-1)^{b+1} \log \mathbf{S}_{b}(x)$ $(b \geq 1)$, the change of variable $x \mapsto 1-x$ gives

$$
I_{p, q}(\boldsymbol{a} ; \boldsymbol{b})=(-1)^{|\boldsymbol{a}|+|\boldsymbol{b}|+q} I_{p, q}(\boldsymbol{a} ; \boldsymbol{b}) .
$$

Hence, the assertion holds.

Proposition 2.2. Let $p$ and $q$ be non-negative integers. Suppose that $a_{1}, \ldots, a_{p} \geq 1$ and $b_{1}, \ldots, b_{q} \geq 2$. Put $\boldsymbol{a}=\left(a_{1}, \ldots, a_{p}\right)$ and $\boldsymbol{b}=\left(b_{1}, \ldots, b_{q}\right)$. If $|\boldsymbol{a}|+|\boldsymbol{b}|+q-1$ is even, then

$$
\sum_{k=1}^{p} a_{k} I_{p, q}\left(\boldsymbol{a}-\boldsymbol{e}_{k}^{(p)} ; \boldsymbol{b}\right)+\sum_{k=1}^{q} b_{k} I_{p, q}\left(\boldsymbol{a} ; \boldsymbol{b}-\boldsymbol{e}_{k}^{(q)}\right)=-2 \prod_{j=1}^{p} B_{a_{i}} \prod_{j=1}^{q} b_{j} \log \mathbf{S}_{b_{j}}(0),
$$

where the row vector $\boldsymbol{e}_{k}^{(r)}$ of dimension $r$ has components 0 except for its $k$-th component, which is equal to 1.

Proof. Note that $\frac{d}{d x} B_{a}(x)=a B_{a-1}(x)(a \geq 1)$ and $\frac{d}{d x} \log \mathbf{S}_{b}(x)=(b-1) \log \mathbf{S}_{b-1}(x)$ $(b \geq 2)$. Hence, by integration by parts, we obtain the formula.

Proposition 2.3. Let $r \in \mathbb{Z}_{\geq 1}$ and $\boldsymbol{c}=\left(c_{1}, \ldots, c_{r}\right) \in\left(\mathbb{Z}_{\geq 1}\right)^{r}$. Then

$$
\sum_{k=0}^{r} \sum_{\substack{K \subset N_{r} \\|K|=k}}(\pi i)^{k} I_{k, r-k}\left(\boldsymbol{c}_{K} ; \boldsymbol{c}_{N_{r} \backslash K}\right)=0 .
$$

Proof. We consider the integral

$$
I_{\varepsilon}:=\int_{\varepsilon}^{1-\varepsilon} \prod_{j=1}^{r} \operatorname{Li}_{c_{j}}\left(e^{2 \pi i x}\right) d x
$$

for $\varepsilon \in(0,1 / 2)$, where $\operatorname{Li}_{s}(z)$ is the polylogarithm. We see that

$$
\begin{aligned}
I_{\varepsilon} & =\int_{\varepsilon}^{1-\varepsilon} \lim _{N \rightarrow \infty} \sum_{n_{1}, \ldots, n_{r}=1}^{N} \frac{e^{2 \pi i x\left(n_{1}+\cdots+n_{r}\right)}}{n_{1}^{c_{1}} \cdots n_{r}^{c_{r}}} d x \\
& =-\frac{1}{\pi} \lim _{N \rightarrow \infty} \sum_{n_{1}, \ldots, n_{r}=1}^{N} \frac{\sin \left(2 \pi\left(n_{1}+\cdots+n_{r}\right) \varepsilon\right)}{n_{1}^{c_{1}} \cdots n_{r}^{c_{r}}\left(n_{1}+\cdots+n_{r}\right)} \\
& \rightarrow 0 \quad(\varepsilon \rightarrow 0) .
\end{aligned}
$$

Here, the second equality and the limit are justified by uniform convergence in $x$ and $\varepsilon$, respectively. Since

$$
\operatorname{Li}_{n}\left(e^{2 \pi i x}\right)=-\frac{(2 \pi i)^{n-1}}{n !}\left(n \log \mathbf{S}_{n}(x)+\pi i B_{n}(x)\right)
$$

for $n \in \mathbb{Z}_{\geq 1}$ and $x \in(0,1)$, we have

$$
\int_{0}^{1} \prod_{j=1}^{r}\left(c_{j} \log \mathbf{S}_{c_{j}}(x)+\pi i B_{c_{j}}(x)\right) d x=0 .
$$

Expanding the product on the left hand side, we deduce the result. 
Remark 2.1. Comparing the real and imaginary parts of both sides of (2.1), we obtain the formulas

$$
\sum_{\substack{k=0 \\ k \text { even }}}^{r} \sum_{\substack{K \subset N_{r} \\|K|=k}}(-1)^{\frac{k}{2}} \pi^{k} I_{k, r-k}\left(\boldsymbol{c}_{K} ; \boldsymbol{c}_{N_{r} \backslash K}\right)=0
$$

and

$$
\sum_{\substack{k=1 \\ k: \text { odd }}}^{r} \sum_{\substack{K \subset N_{r} \\|K|=k}}(-1)^{\frac{k-1}{2}} \pi^{k-1} I_{k, r-k}\left(\boldsymbol{c}_{K} ; \boldsymbol{c}_{N_{r} \backslash K}\right)=0 .
$$

We now prove a lemma needed later.

Lemma 2.4. Let $p$ be a positive integer and $a_{1}, \ldots, a_{p}$ be non-negative integers. Put $\boldsymbol{a}=\left(a_{1}, \ldots, a_{p}\right)$. Then

$$
\prod_{i=1}^{p} B_{a_{i}}(x)=\sum_{l} \mathcal{B}_{p}(\boldsymbol{a} ; l) B_{|\boldsymbol{a}|-2 l}(x)+I_{p, 0}(\boldsymbol{a})
$$

where the sum is taken over all integers $l \in[0,|\boldsymbol{a}| / 2)$. Here

$$
\mathcal{B}_{p}(\boldsymbol{a} ; l):=\frac{-2}{|\boldsymbol{a}|-2 l} \sum_{\substack{l_{1}, \ldots, l_{p} \geq 0 \\
l_{1}+\cdots+l_{p}=2 l+1}} \prod_{j=1}^{p}\left(\begin{array}{l}
a_{j} \\
l_{j}
\end{array}\right) B_{l_{j}}
$$

for an integer $l \in[0,|\boldsymbol{a}| / 2)$.

Proof. We expand, in two ways, the function

$$
\Phi_{p}(x ; \boldsymbol{u})=\prod_{j=1}^{p} \frac{u_{j} e^{x u_{j}}}{e^{u_{j}}-1} \quad\left(\boldsymbol{u}=\left(u_{1}, \ldots, u_{p}\right)\right)
$$

as a power series in the variables $u_{1}, \ldots, u_{p}$. In the first way, we directly use the definition of the Bernoulli polynomial to obtain

$$
\Phi_{p}(x ; \boldsymbol{u})=\sum_{a_{1}, \ldots, a_{p}=0}^{\infty} B_{a_{1}}(x) \cdots B_{a_{p}}(x) \frac{u_{1}^{a_{1}} \cdots u_{p}^{a_{p}}}{a_{1} ! \cdots a_{p} !}
$$

when $\left|u_{1}\right|, \ldots,\left|u_{p}\right|<2 \pi$. In the second way, we apply the equation $\Phi_{p}(x ; \boldsymbol{u})=|\boldsymbol{u}|^{-1}\left(\Phi_{1}(x ;|\boldsymbol{u}|)-1\right)\left(\Phi_{p}(0 ;-\boldsymbol{u})-\Phi_{p}(0 ; \boldsymbol{u})\right)+|\boldsymbol{u}|^{-1}\left(e^{|\boldsymbol{u}|}-1\right) \Phi_{p}(0 ; \boldsymbol{u})$.

By simple calculation, the first term on the right hand side is equal to

$$
\sum_{a_{1}, \ldots, a_{p}=0}^{\infty}\left(\sum_{l} \mathcal{B}_{p}(\boldsymbol{a} ; l) B_{|\boldsymbol{a}|-2 l}(x)\right) \frac{u_{1}^{a_{1}} \cdots u_{p}^{a_{p}}}{a_{1} ! \cdots a_{p} !}
$$

when $\left|u_{1}\right|, \ldots,\left|u_{p}\right|,|\boldsymbol{u}|<2 \pi$, where $l$ runs over all integers in $[0,|\boldsymbol{a}| / 2)$. The second term is equal to

$$
\int_{0}^{1} \Phi_{p}(t ; \boldsymbol{u}) d t=\sum_{a_{1}, \ldots, a_{p}=0}^{\infty} I_{p, 0}(\boldsymbol{a}) \frac{u_{1}^{a_{1}} \cdots u_{p}^{a_{p}}}{a_{1} ! \cdots a_{p} !}
$$


when $\left|u_{1}\right|, \ldots,\left|u_{p}\right|<2 \pi$. Here, term-by-term integration is justified by uniform convergence for $t \in(0,1)$. Hence

$$
\Phi_{p}(x ; \boldsymbol{u})=\sum_{a_{1}, \ldots, a_{p}=0}^{\infty}\left(\sum_{l} \mathcal{B}_{p}(\boldsymbol{a} ; l) B_{|\boldsymbol{a}|-2 l}(x)+I_{p, 0}(\boldsymbol{a})\right) \frac{u_{1}^{a_{1}} \cdots u_{p}^{a_{p}}}{a_{1} ! \cdots a_{p} !}
$$

when $\left|u_{1}\right|, \ldots,\left|u_{p}\right|,|\boldsymbol{u}|<2 \pi$. Thus, comparing the coefficients of $u_{1}^{a_{1}} \cdots u_{p}^{a_{p}}$ in (2.5) and (2.6), we obtain formula (2.4).

Proposition 2.5. Let $p$ be a positive integer $\geq 2$ and let $\boldsymbol{a}=\left(a_{1}, \ldots, a_{p}\right) \in\left(\mathbb{Z}_{\geq 1}\right)^{p}$. Put $\boldsymbol{a}_{p}=\left(a_{1}, \ldots, a_{p-1}\right)$. Then, we have $I_{1,0}(a)=0$ for an integer $a$, and

$$
I_{p, 0}(\boldsymbol{a})=(-1)^{a_{p}+1} a_{p} ! \sum_{l} \mathcal{B}_{p-1}\left(\boldsymbol{a}_{p} ; l\right) \frac{\left(\left|\boldsymbol{a}_{p}\right|-2 l\right) !}{(|\boldsymbol{a}|-2 l) !} B_{|\boldsymbol{a}|-2 l}
$$

where the sum is taken over all integers $l \in\left[0,\left|\boldsymbol{a}_{p}\right| / 2\right)$.

Proof. The first assertion is trivial. If $p=2$, we can show it by integration by parts. When $p \geq 3$, it holds by applying (2.4) to $B_{a_{1}}(x) \cdots B_{a_{p-1}}(x)$.

Remark 2.2. The result for $I_{2,0}$ was given by Nörlund [28, p.31] and by Mordell [26, p.375], and $I_{3,0}$ was calculated by Carlitz [3. The methods of Mordell and Carlitz can be found in [2, p.276].

Proposition 2.6. Let $p, q, r$ be integers with $p \geq 1$ and $q, r \geq 0$. Let $\boldsymbol{a}=$ $\left(a_{1}, \ldots, a_{p}\right)$ and $\boldsymbol{b}=\left(b_{1}, \ldots, b_{q}\right)$, whose components are non-negative integers, and let $\boldsymbol{c}=\left(c_{1}, \ldots, c_{r}\right) \in\left(\mathbb{Z}_{\geq 1}\right)^{r}$. Then

$$
I_{p+q, r}(\boldsymbol{a}, \boldsymbol{b} ; \boldsymbol{c})=\sum_{l} \mathcal{B}_{p}(\boldsymbol{a} ; l) I_{q+1, r}(|\boldsymbol{a}|-2 l, \boldsymbol{b} ; \boldsymbol{c})+I_{p, 0}(\boldsymbol{a}) I_{q, r}(\boldsymbol{b} ; \boldsymbol{c}),
$$

where the sum is taken over all integers $l \in[0,|\boldsymbol{a}| / 2)$.

Proof. The result immediately follows from (2.4).

\section{The Mordell-Tornheim Zeta values}

In 1957, Mordell proved that

$$
\zeta_{M T, 2}(a, a ; a)=(-1)^{a / 2-1} \frac{(2 \pi)^{3 a}}{6(a !)^{3}} \int_{0}^{1} B_{a}(x)^{3} d x
$$

for an even integer $a \geq 2$. This fact can be found in [25, p.371], though his definition of the Bernoulli polynomial is slightly different from ours. Employing the generalized $\log$ sine integral $I_{p, q}$, we can formulate his result in the general cases.

Theorem 1. Let $r$ be a positive integer and let $\boldsymbol{c}=\left(c_{1}, \ldots, c_{r+1}\right) \in\left(\mathbb{Z}_{\geq 1}\right)^{r+1}$. Then,

$$
\begin{aligned}
\zeta_{M T, r}\left(c_{1}, \ldots, c_{r} ; c_{r+1}\right)= & \frac{(-1)^{c_{r+1}+r+1}(2 \pi i)^{|\boldsymbol{c}|-r}}{c_{1} ! \cdots c_{r+1} !} \\
& \times \sum_{k=0}^{r} \sum_{\substack{K \subset N_{r} \\
|K|=k}}(\pi i)^{k} I_{k+1, r-k}\left(c_{r+1}, \boldsymbol{c}_{K} ; \boldsymbol{c}_{N_{r} \backslash K}\right) .
\end{aligned}
$$


Proof. We consider the integral

$$
\int_{0}^{1} \operatorname{Li}_{c_{r+1}}\left(e^{-2 \pi i x}\right) \prod_{j=1}^{r} \operatorname{Li}_{c_{j}}\left(e^{2 \pi i x}\right) d x .
$$

In the same way as in the proof of Proposition 2.3, we have

$$
\begin{aligned}
& (-1)^{c_{r+1}+r} \frac{c_{1} ! \cdots c_{r+1} !}{(2 \pi i)^{|c|-r-1}} \zeta_{M T, r}\left(c_{1}, \ldots, c_{r} ; c_{r+1}\right) \\
& =\int_{0}^{1}\left(c_{r+1} \log \mathbf{S}_{c_{r+1}}(x)-\pi i B_{c_{r+1}}(x)\right) \prod_{j=1}^{r}\left(c_{j} \log \mathbf{S}_{c_{j}}(x)+\pi i B_{c_{j}}(x)\right) d x .
\end{aligned}
$$

Proposition 2.3 shows that the right hand side is equal to

$$
-2 \pi i \int_{0}^{1} B_{c_{r+1}}(x) \prod_{j=1}^{r}\left(c_{j} \log \mathbf{S}_{c_{j}}(x)+\pi i B_{c_{j}}(x)\right) d x .
$$

Thus, we obtain (3.10).

Remark 3.1. Comparing the real parts of both sides of (3.10), we obtain the following. If $|\boldsymbol{c}|+r$ is odd, then

$$
\begin{aligned}
\zeta_{M T, r}\left(c_{1}, \ldots, c_{r} ; c_{r+1}\right)= & \frac{(-1)^{\frac{|c|+r-1}{2}+c_{r+1}(2 \pi)^{|c|-r+1}}}{2 c_{1} ! \cdots c_{r+1} !} \\
& \times \sum_{\substack{k=1 \\
k: \text { odd }}}^{r} \sum_{\substack{K \subset N_{r} \\
|K|=k}}(-1)^{\frac{k-1}{2}} \pi^{k-1} I_{k+1, r-k}\left(c_{r+1}, \boldsymbol{c}_{K} ; \boldsymbol{c}_{N_{r} \backslash K}\right),
\end{aligned}
$$

and if $|\boldsymbol{c}|+r$ is even, then

$$
\begin{aligned}
\zeta_{M T, r}\left(c_{1}, \ldots, c_{r} ; c_{r+1}\right)= & \frac{(-1)^{\frac{|c|+r-2}{2}+c_{r+1}(2 \pi)^{|c|-r}}}{c_{1} ! \cdots c_{r+1} !} \\
& \times \sum_{\substack{k=0 \\
k \text { :even }}}^{r} \sum_{\substack{K \subset N_{r} \\
|K|=k}}(-1)^{\frac{k}{2}} \pi^{k} I_{k+1, r-k}\left(c_{r+1}, \boldsymbol{c}_{K} ; \boldsymbol{c}_{N_{r} \backslash K}\right) .
\end{aligned}
$$

Remark 3.2. There is preceding research [5, 23, 27, 30, 31] for the expression of the Mordell-Tornheim zeta function by integrals involving the polylogarithms or associated functions. For other expressions, refer to [11, 21, 22. Moreover, in [12, we can find integral expressions of the multi-variable Witten zeta-function which can be regarded as a generalization of the Mordell-Tornheim zeta function.

Theorem 1 exhibits the Mordell-Tornheim zeta values as sums of generalized log sine integrals. In order to prove the converse of this assertion, we first recall the Euler numbers $E_{n}\left(n \in \mathbb{Z}_{\geq 0}\right)$ defined as

$$
\frac{1}{\cosh t}=\sum_{n=0}^{\infty} \frac{E_{n}}{n !} t^{n} \quad(|t|<\pi / 2) .
$$


It is clear that $E_{n}=0$ if $n$ is odd. We need two well-known formulas: for a non-negative integer $n$,

$$
\sum_{k=0}^{n}\left(\begin{array}{l}
2 n \\
2 k
\end{array}\right) E_{2 k}= \begin{cases}1 & \text { if } n=0 \\
0 & \text { otherwise }\end{cases}
$$

and for a positive integer $n$,

$$
\sum_{k=0}^{n-1}\left(\begin{array}{c}
2 n-1 \\
2 k
\end{array}\right) E_{2 k}=\frac{2^{2 n}\left(2^{2 n}-1\right) B_{2 n}}{2 n} .
$$

By the above properties of the Euler numbers, we obtain the following expression of the integral $I_{1, q}$.

Theorem 2. Let $q$ be a positive integer. Suppose that $a \in \mathbb{Z}_{\geq 0}$ and $b_{1}, \ldots, b_{q} \in \mathbb{Z}_{\geq 1}$. Put $\boldsymbol{b}=\left(b_{1}, \ldots, b_{q}\right)$. If $a+|\boldsymbol{b}|+q$ is even, then

$$
\begin{aligned}
& I_{1, q}(a ; \boldsymbol{b}) \\
& =\frac{(-1)^{\frac{a-|\boldsymbol{b}|-q-2}{2}}}{(2 \pi)^{a+|\boldsymbol{b}|-q}} \sum_{\substack{k=0 \\
k: \text { even }}}^{q-1} \frac{E_{k}}{2^{k}} \sum_{\substack{K \subset N_{q} \\
|K|=k}} \sum_{l}(-1)^{\left|\boldsymbol{b}_{K}\right|+l}\left(a+\left|\boldsymbol{b}_{K}\right|-2 l\right) !\left(\prod_{j \in N_{q} \backslash K} b_{j} !\right) \\
& \quad \times(2 \pi)^{2 l} \mathcal{B}_{k+1}\left(a, \boldsymbol{b}_{K} ; l\right) \zeta_{M T, q-k}\left(\boldsymbol{b}_{N_{q} \backslash K} ; a+\left|\boldsymbol{b}_{K}\right|-2 l\right) \\
& \quad+(-1)^{\frac{q}{2}} \pi^{q} E_{q} I_{q+1,0}(a, \boldsymbol{b}),
\end{aligned}
$$

where $l$ runs over all integers in $\left[0,\left(a+\left|\boldsymbol{b}_{K}\right|\right) / 2\right)$.

We prove two lemmas which will be useful in proving Theorems 2, 3, and 4.

Lemma 3.1. Let $p, q$ be positive integers. Let $\boldsymbol{a}=\left(a_{1}, \ldots, a_{p}\right) \in\left(\mathbb{Z}_{\geq 0}\right)^{p}$ and $\boldsymbol{b}=\left(b_{1}, \ldots, b_{q}\right) \in\left(\mathbb{Z}_{\geq 1}\right)^{q}$. If $|\boldsymbol{a}|+|\boldsymbol{b}|+q$ is even, then

$$
\begin{aligned}
& \sum_{0 \leq l<|\boldsymbol{a}| / 2} \mathcal{B}_{p}(\boldsymbol{a} ; l) \hat{\zeta}_{M T, q}(\boldsymbol{b} ;|\boldsymbol{a}|-2 l) \\
& =\sum_{\substack{k=0 \\
k: \text { even }}} \sum_{\substack{K \subset N_{q} \\
|K|=k}}(-1)^{\frac{k}{2}} \pi^{k} I_{p+k, q-k}\left(\boldsymbol{a}, \boldsymbol{b}_{K} ; \boldsymbol{b}_{N_{q} \backslash K}\right),
\end{aligned}
$$

where, for $\boldsymbol{c}=\left(c_{1}, \ldots, c_{r+1}\right) \in\left(\mathbb{Z}_{\geq 1}\right)^{r+1}$ with $|\boldsymbol{c}|+r$ even,

$$
\hat{\zeta}_{M T, r}\left(c_{1}, \ldots, c_{r} ; c_{r+1}\right):=\frac{(-1)^{\frac{|c|+r-2}{2}+c_{r+1}} c_{1} ! \cdots c_{r+1} !}{(2 \pi)^{|c|-r}} \zeta_{M T, r}\left(c_{1}, \ldots, c_{r} ; c_{r+1}\right) .
$$

Proof. If $a_{1}=\cdots=a_{p}=0$, then formula (3.15) coincides with (2.2). In the other cases, applying formulas (3.12), (2.8) and (2.2) to the left hand side of (3.15) in order, we can show that it coincides with the right hand side. Thus, formula (3.15) holds.

Lemma 3.2. Let $A=\left\{a_{i j}\right\}$ be a square matrix. If $A=\left\{\left(\begin{array}{c}2 j-2 \\ 2 i-2\end{array}\right)\right\}$ or $\left\{\left(\begin{array}{c}2 j-1 \\ 2 i-1\end{array}\right)\right\}$, then $A^{-1}=\left\{a_{i j} E_{2(j-i)}\right\}$.

Proof. The result follows from (3.13). 
Proof of Theorem 2, Put

$$
A=\left\{\left(\begin{array}{l}
2 j-2 \\
2 i-2
\end{array}\right)\right\}(1 \leq i, j \leq\lfloor q / 2\rfloor+1) .
$$

Set $X={ }^{t}\left(X_{0}, X_{2}, \ldots, X_{2\lfloor q / 2\rfloor}\right)$ and $Y={ }^{t}\left(Y_{0}, Y_{2}, \ldots, Y_{2\lfloor q / 2\rfloor}\right)$ whose components satisfy

$$
\begin{aligned}
X_{k} & =(-1)^{\frac{k}{2}} \pi^{k} \sum_{\substack{K \subset N_{q} \\
|K|=k}} I_{k+1, q-k}\left(a, \boldsymbol{b}_{K} ; \boldsymbol{b}_{N_{q} \backslash K}\right), \\
Y_{k} & =(-1)^{\frac{k}{2}} \pi^{k} \sum_{\substack{K \subset N_{q} \\
|K|=k}} \sum_{l} \mathcal{B}_{k+1}\left(a, \boldsymbol{b}_{K} ; l\right) \hat{\zeta}_{M T, q-k}\left(\boldsymbol{b}_{N_{q} \backslash K} ; a+\left|\boldsymbol{b}_{K}\right|-2 l\right)
\end{aligned}
$$

for any even integer $k \in[0, q)$, and $X_{q}=Y_{q}=(-1)^{\frac{q}{2}} \pi^{q} I_{q+1,0}(a, b)$ if $q$ is even. Then, Lemma 3.1 shows $A X=Y$. Hence, by Lemma 3.2, we have

$$
I_{1, q}(a ; \boldsymbol{b})=X_{0}=\sum_{\substack{k=0 \\ k: \text { even }}}^{q} E_{k} Y_{k} .
$$

This completes the proof of Theorem 2

Modifying the proof of Theorem 2, we gain two results for relations among the Mordell-Tornheim zeta values. The first result is an explicit formula for the parity result of Tsumura [34, Theorem 1.1] that the zeta value $\zeta_{M T, r}\left(c_{1}, \ldots, c_{r} ; c_{r+1}\right)$ $\left(c_{1}, \ldots, c_{r+1} \in \mathbb{Z}_{\geq 1}\right)$ can be expressed as a rational linear combination of products of values of $\zeta_{M T, q}(q<r)$ at positive integers if $r$ and $\sum_{j=1}^{r+1} c_{j}$ are of different parity. Here, we remark that $\pi^{2 l}\left(l \in \mathbb{Z}_{\geq 1}\right)$ is a rational multiple of $\zeta(2 l)=\zeta_{M T, 1}(l ; l)$.

Theorem 3. Let $r$ be a positive integer and $\boldsymbol{c}=\left(c_{1}, \ldots, c_{r+1}\right) \in\left(\mathbb{Z}_{\geq 1}\right)^{r+1}$. If $|\boldsymbol{c}|+r$ is odd, then

$$
\begin{aligned}
& \zeta_{M T, r}\left(c_{1}, \ldots, c_{r} ; c_{r+1}\right) \\
& =2 \sum_{\substack{k=1 \\
k: \text { odd }}}^{r-1} \frac{2^{k+1}-1}{k+1} B_{k+1} \sum_{\substack{K \subset N_{r} \\
|K|=k}} \sum_{l} \frac{(-1)^{\left|\boldsymbol{c}_{K}\right|+l+1}\left(\left|\boldsymbol{c}_{K}\right|+c_{r+1}-2 l\right) !}{c_{r+1} ! \prod_{j \in K} c_{j} !} \\
& \quad \times(2 \pi)^{2 l} \mathcal{B}_{k+1}\left(c_{r+1}, \boldsymbol{c}_{K} ; l\right) \zeta_{M T, r-k}\left(\boldsymbol{c}_{N_{r} \backslash K} ;\left|\boldsymbol{c}_{K}\right|+c_{r+1}-2 l\right) \\
& \quad+(-1)^{\frac{|\boldsymbol{c}|}{2}+c_{r+1}} \frac{2\left(2^{r+1}-1\right)}{r+1} B_{r+1} \frac{(2 \pi)^{|\boldsymbol{c}|}}{c_{1} ! \cdots c_{r+1} !} I_{r+1,0}(\boldsymbol{c}),
\end{aligned}
$$

where $l$ runs over all integers in $\left[0,\left(\left|\boldsymbol{c}_{K}\right|+c_{r+1}\right) / 2\right)$.

Proof. Put

$$
A=\left\{\left(\begin{array}{l}
2 j-1 \\
2 i-1
\end{array}\right)\right\}(1 \leq i, j \leq\lfloor(r+1) / 2\rfloor)
$$


Set $X={ }^{t}\left(X_{1}, X_{3}, \ldots, X_{2\lfloor(r+1) / 2\rfloor-1}\right)$ and $Y={ }^{t}\left(Y_{1}, Y_{3}, \ldots, Y_{2\lfloor(r+1) / 2\rfloor-1}\right)$ whose components satisfy

$$
\begin{aligned}
X_{k} & =(-1)^{\frac{k-1}{2}} \pi^{k-1} \sum_{\substack{K \subset N_{r} \\
|K|=k}} I_{k+1, r-k}\left(c_{r+1}, \boldsymbol{c}_{K} ; \boldsymbol{c}_{N_{r} \backslash K}\right), \\
Y_{k} & =(-1)^{\frac{k-1}{2}} \pi^{k-1} \sum_{\substack{K \subset N_{r} \\
|K|=k}} \sum_{l} \mathcal{B}_{k+1}\left(c_{r+1}, \boldsymbol{c}_{K} ; l\right) \hat{\zeta}_{M T, r-k}\left(\boldsymbol{c}_{N_{r} \backslash K} ; c_{r+1}+\left|\boldsymbol{c}_{K}\right|-2 l\right)
\end{aligned}
$$

for any odd integer $k \in[1, r)$, and $X_{r}=Y_{r}=(-1)^{\frac{r-1}{2}} \pi^{r-1} I_{r+1,0}(\boldsymbol{c})$ if $r$ is odd. Then, Lemma 3.1 shows $A X=Y$. Hence, by Lemma 3.2 and formula (3.14), we have

$$
\sum_{\substack{k=1 \\ k: \text { odd }}}^{r} X_{k}=\sum_{\substack{k=1 \\ k: \text { odd }}}^{r} \frac{2^{k+1}\left(2^{k+1}-1\right)}{k+1} B_{k+1} Y_{k} .
$$

Thus, we conclude Theorem 3 by (3.11).

Example 3.1. (i) Let $c_{1}, c_{2}, c_{3}$ be positive integers. If $c_{1}+c_{2}+c_{3}$ is odd, then

$$
\zeta_{M T, 2}\left(c_{1}, c_{2} ; c_{3}\right)=(-1)^{c_{1}} J_{2,1}\left(c_{1}, c_{3} ; c_{2}\right)+(-1)^{c_{2}} J_{2,1}\left(c_{2}, c_{3} ; c_{1}\right) .
$$

Here, for positive integers $a_{1}, a_{2}, a_{3}$,

$$
\begin{aligned}
J_{2,1}\left(a_{1}, a_{2} ; a_{3}\right)= & \sum_{k}\left\{\left(\begin{array}{c}
a_{1}+a_{2}-1-2 k \\
a_{1}-1
\end{array}\right)+\left(\begin{array}{c}
a_{1}+a_{2}-1-2 k \\
a_{2}-1
\end{array}\right)\right\} \\
& \times \zeta(2 k) \zeta\left(a_{1}+a_{2}+a_{3}-2 k\right),
\end{aligned}
$$

where the sum is taken over all integers $k \in\left[0, \max \left\{a_{1}, a_{2}\right\} / 2\right]$. This is properly included in the result of Huard-Williams-Zhang [10, Theorem 2]. They proved a formula available for $c_{1}, c_{2} \in \mathbb{Z}_{\geq 0}$ and $c_{3} \in \mathbb{Z}_{\geq 1}$ satisfying $c_{1}+c_{2} \geq 1, c_{1}+c_{3} \geq 2$, and $c_{2}+c_{3} \geq 2$, which equals (3.16) in our case.

(ii) Let $c_{1}, c_{2}, c_{3}, c_{4}$ be positive integers. If $c_{1}+c_{2}+c_{3}+c_{4}$ is even, then

$$
\begin{aligned}
\zeta_{M T, 3}\left(c_{1}, c_{2}, c_{3} ; c_{4}\right) \\
=(-1)^{c_{1}} J_{2,2}\left(c_{1}, c_{4} ; c_{2}, c_{3}\right)+(-1)^{c_{2}} J_{2,2}\left(c_{2}, c_{4} ; c_{3}, c_{1}\right)+(-1)^{c_{3}} J_{2,2}\left(c_{3}, c_{4} ; c_{1}, c_{2}\right) \\
\quad+(-1)^{\frac{c_{1}+c_{2}+c_{3}-c_{4}}{2}}+1 \frac{(2 \pi)^{c_{1}+c_{2}+c_{3}+c_{4}}}{4 c_{1} ! c_{2} ! c_{3} ! c_{4} !} I_{4,0}\left(c_{1}, c_{2}, c_{3}, c_{4}\right) .
\end{aligned}
$$

Here, we set, for positive integers $a_{1}, a_{2}, a_{3}, a_{4}$,

$$
\begin{aligned}
J_{2,2}\left(a_{1}, a_{2} ; a_{3}, a_{4}\right)= & \sum_{k}\left\{\left(\begin{array}{c}
a_{1}+a_{2}-1-2 k \\
a_{1}-1
\end{array}\right)+\left(\begin{array}{c}
a_{1}+a_{2}-1-2 k \\
a_{2}-1
\end{array}\right)\right\} \\
& \times \zeta(2 k) \zeta_{M T, 2}\left(a_{3}, a_{4} ; a_{1}+a_{2}-2 k\right),
\end{aligned}
$$

where the sum is taken over all integers $k \in\left[0, \max \left\{a_{1}, a_{2}\right\} / 2\right]$. An expression of $I_{4,0}\left(c_{1}, c_{2}, c_{3}, c_{4}\right)$ by the Riemann zeta values will be described in Example 4.3 . Formula (3.17) seems simpler than the result of Matsumoto-Nakamura-Ochiai-Tsumura [23, Theorem 4.6].

Next, we show another relation for the Mordell-Tornheim zeta values. 
Theorem 4. Let $r \in \mathbb{Z}_{\geq 1}$ and $\boldsymbol{c}=\left(c_{1}, \ldots, c_{r+1}\right) \in\left(\mathbb{Z}_{\geq 1}\right)^{r}$. If $|\boldsymbol{c}|+r$ is even, then

$$
\begin{aligned}
\sum_{\substack{k=1 \\
k: \text { odd }}}^{r} & \frac{2^{k+1}-1}{k+1} B_{k+1} \sum_{\substack{K \subset N_{r+1} \\
|K|=k}} \sum_{l} \frac{(-1)^{\left|\boldsymbol{c}_{K}\right|+l}\left(\left|\boldsymbol{c}_{K}\right|-2 l\right) !}{\prod_{j \in K} c_{j} !} \\
\quad & \times(2 \pi)^{2 l} \mathcal{B}_{k}\left(\boldsymbol{c}_{K} ; l\right) \zeta_{M T, r+1-k}\left(\boldsymbol{c}_{N_{r+1} \backslash K} ;\left|\boldsymbol{c}_{K}\right|-2 l\right) \\
\quad & (-1)^{\frac{|c|}{2}} \frac{2^{r+2}-1}{r+2} B_{r+2} \frac{(2 \pi)^{|\boldsymbol{c}|}}{c_{1} ! \cdots c_{r+1} !} I_{r+1,0}(\boldsymbol{c}),
\end{aligned}
$$

where $l$ runs over all integers in $\left[0,\left|\boldsymbol{c}_{K}\right| / 2\right)$.

Proof. Put $A=\left\{\left(\begin{array}{c}2 j-1 \\ 2 i-1\end{array}\right)\right\}(1 \leq i, j \leq\lfloor r / 2\rfloor+1)$. Set $X={ }^{t}\left(X_{1}, X_{3}, \ldots, X_{2\lfloor r / 2\rfloor+1}\right)$ and $Y={ }^{t}\left(Y_{1}, Y_{3}, \ldots, Y_{2\lfloor r / 2\rfloor+1}\right)$ whose components satisfy

$$
\begin{aligned}
X_{k} & =(-1)^{\frac{k-1}{2}} \pi^{k-1} \sum_{\substack{K \subset N_{r+1} \\
|K|=k}} I_{k, r+1-k}\left(\boldsymbol{c}_{K} ; \boldsymbol{c}_{N_{r+1} \backslash K}\right), \\
Y_{k} & =(-1)^{\frac{k-1}{2}} \pi^{k-1} \sum_{\substack{K \subset N_{r+1} \\
|K|=k}} \sum_{l} \mathcal{B}_{k}\left(\boldsymbol{c}_{K} ; l\right) \hat{\zeta}_{M T, r+1-k}\left(\boldsymbol{c}_{N_{r+1} \backslash K} ;\left|\boldsymbol{c}_{K}\right|-2 l\right)
\end{aligned}
$$

for any odd integer $k \in[1, r+1)$, and $X_{r+1}=Y_{r+1}=(-1)^{\frac{r}{2}} \pi^{r} I_{r+1,0}(\boldsymbol{c})$ if $r$ is even. Then, in the same way as in the proof of Theorem 3, we have $A X=Y$ and

$$
\sum_{\substack{k=1 \\ k: \text { odd }}}^{r+1} X_{k}=\sum_{\substack{k=1 \\ k \text { :odd }}}^{r+1} \frac{2^{k+1}\left(2^{k+1}-1\right)}{k+1} B_{k+1} Y_{k} .
$$

Since the left hand side vanishes by (2.3), we obtain the result.

Example 3.2. (i) If $c_{1}+c_{2}+c_{3}$ is even, then

$$
\begin{aligned}
& \zeta_{M T, 2}\left(c_{1}, c_{2} ; c_{3}\right)+(-1)^{c_{2}} \zeta_{M T, 2}\left(c_{2}, c_{3} ; c_{1}\right)+(-1)^{c_{1}} \zeta_{M T, 2}\left(c_{3}, c_{1} ; c_{2}\right) \\
& \quad=2 J_{2,1}\left(c_{1}, c_{2} ; c_{3}\right) .
\end{aligned}
$$

This is a special case of the result by Nakamura [27, Theorem 1.2]. He proved that this formula holds for $c_{1}, c_{2} \in \mathbb{Z}_{\geq 1}$ and $c_{3} \in \mathbb{C}$ except for the singular points. Before his work, Tsumura [35. Theorem 4.5] had given a functional relation which is complicated but equivalent to Nakamura's result (see [23, p.102]). Other studies before them are found in [31, 33].

(ii) If $c_{1}+c_{2}+c_{3}+c_{4}$ is odd, then

$$
\begin{aligned}
& (-1)^{c_{4}} \zeta_{M T, 3}\left(c_{1}, c_{2}, c_{3} ; c_{4}\right)+(-1)^{c_{1}} \zeta_{M T, 3}\left(c_{2}, c_{3}, c_{4} ; c_{1}\right) \\
& +(-1)^{c_{2}} \zeta_{M T, 3}\left(c_{3}, c_{4}, c_{1} ; c_{2}\right)+(-1)^{c_{3}} \zeta_{M T, 3}\left(c_{4}, c_{1}, c_{2} ; c_{3}\right) \\
& \quad=(-1)^{c_{4}} J_{3,1}\left(c_{1}, c_{2}, c_{3} ; c_{4}\right)+(-1)^{c_{1}} J_{3,1}\left(c_{2}, c_{3}, c_{4} ; c_{1}\right) \\
& \quad+(-1)^{c_{2}} J_{3,1}\left(c_{3}, c_{4}, c_{1} ; c_{2}\right)+(-1)^{c_{3}} J_{3,1}\left(c_{4}, c_{1}, c_{2} ; c_{3}\right),
\end{aligned}
$$


where, for positive integers $a_{1}, a_{2}, a_{3}, a_{4}$,

$$
\begin{aligned}
J_{3,1}\left(a_{1}, a_{2}, a_{3} ; a_{4}\right) & \\
:= & 3\left(\begin{array}{c}
a_{1}+a_{2}+a_{3}-3 \\
a_{1}-1, a_{2}-1, a_{3}-1
\end{array}\right) \zeta(2) \zeta\left(a_{1}+a_{2}+a_{3}+a_{4}-2\right) \\
& -2 \sum_{k, l}\left\{\left(\begin{array}{c}
a_{1}+a_{2}+a_{3}-1-2 k-2 l \\
a_{1}-1, a_{2}-2 k, a_{3}-2 l
\end{array}\right)+\left(\begin{array}{c}
a_{1}+a_{2}+a_{3}-1-2 k-2 l \\
a_{1}-2 l, a_{2}-1, a_{3}-2 k
\end{array}\right)\right. \\
& \left.+\left(\begin{array}{c}
a_{1}+a_{2}+a_{3}-1-2 k-2 l \\
a_{1}-2 k, a_{2}-2 l, a_{3}-1
\end{array}\right)\right\} \zeta(2 k) \zeta(2 l) \zeta\left(a_{1}+a_{2}+a_{3}+a_{4}-2 k-2 l\right),
\end{aligned}
$$

where the sum is taken over all non-negative integers $k, l$ such that $k+l<\left(a_{1}+a_{2}+\right.$ $\left.a_{3}\right) / 2$. The corresponding functional relation was proved by Matsumoto-NakamuraOchiai-Tsumura [23, Theorem 4.5]. However, unlike the case of $\zeta_{M T, 2}$, it is different from our formulation.

\section{Relations to the Riemann zeta values}

We investigate some cases in which the integral $I_{p, q}(\boldsymbol{a} ; \boldsymbol{b})$ is described by the Riemann zeta values. As a result, we obtain various relations between the MordellTornheim zeta values and the Riemann zeta values, which contain most of the known results and some new ones.

4.1. Log sine integrals and $\zeta_{M T, r}(1, \ldots, 1 ; a)$. It is known that $I_{1, q}(a ; \mathbf{1})$ with $\mathbf{1}=(1, \ldots, 1)$ can be expressed in terms of the Riemann zeta values (see [19]). In this subsection, the generating function of $I_{1, q}(a ; \mathbf{1})$ becomes clear. Moreover, we show the corresponding results for $\zeta_{M T, r}(1, \ldots, 1 ; a)$.

Proposition 4.1. (i) For $u, v \in \mathbb{C}$ with $|u|<1$ and $|v|<1$,

$$
\sum_{k, l=0}^{\infty}(-1)^{k+l}(2 \pi)^{2 l} I_{1, k}(2 l ; \mathbf{1}) \frac{u^{k} v^{2 l}}{k !(2 l) !}=\frac{\Gamma(1-u) \Gamma(1+v) \Gamma(1-v)}{\Gamma(1-u / 2+v) \Gamma(1-u / 2-v)} .
$$

(ii) For $u, v \in \mathbb{C}$ with $|u|<1$ and $|v|<1$,

$$
1-\sum_{k, l=1}^{\infty} \frac{1}{k !} \zeta_{M T, k}(1, \ldots, 1 ; l) u^{k} v^{l}=\frac{\Gamma(1-u) \Gamma(1-v)}{\Gamma(1-u-v)} .
$$

Proof. (i) We apply the formula

$$
\int_{0}^{1}(2 \sin \pi x)^{\mu} e^{2 \pi i \nu x} d x=\frac{e^{\pi i \nu} \Gamma(1+\mu)}{\Gamma(1+\mu / 2+\nu) \Gamma(1+\mu / 2-\nu)}
$$

for $\mu, \nu \in \mathbb{C}$ with $\operatorname{Re}(\mu)>-1$ [19, (7.136)]. We can check that

$$
\int_{0}^{1}(2 \sin \pi x)^{-u}\left(\frac{2 \pi i v e^{2 \pi i v x}}{e^{2 \pi i v}-1}\right) d x=\frac{\Gamma(1-u) \Gamma(1+v) \Gamma(1-v)}{\Gamma(1-u / 2+v) \Gamma(1-u / 2-v)}
$$

for $u, v \in \mathbb{C}$ with $\operatorname{Re}(u)<1$. We expand the left hand side as a power series to obtain the result. Here, term-by-term integration is justified when $|u|<1$ and $|v|<1$. 
(ii) Formula (4.19) shows

$$
\int_{0}^{1}(2 \sin \pi x)^{-u} e^{-\pi i u B_{1}(x)}\left(\frac{-2 \pi i v e^{-2 \pi i v x}}{e^{-2 \pi i v}-1}\right) d x=\frac{\Gamma(1-u) \Gamma(1-v)}{\Gamma(1-u-v)}
$$

for $u, v \in \mathbb{C}$ with $\operatorname{Re}(u)<1$. When $|u|<1$ and $|v|<1$, we can show that the left hand side is equal to

$$
\sum_{k, l=0}^{\infty}(-1)^{k+l}(2 \pi i)^{l} \frac{u^{k} v^{l}}{k ! l !} \sum_{j=0}^{k}\left(\begin{array}{c}
k \\
j
\end{array}\right)(\pi i)^{j} I_{j+1, k-j}(l, \mathbf{1} ; \mathbf{1}) .
$$

Applying Proposition 2.3 and Theorem 1, we deduce the result.

Remark 4.1. Hoffman gave formula (4.18) by a different method (see the proof of 9. Theorem 5.2]). Moreover, it can also be proved by combining the following two facts.

(i) (Hoffman [8, Corollary 4.2]) For positive integers $r$ and $a$,

$$
\zeta_{M T, r}(1, \ldots, 1 ; a)=r ! \zeta(r+1, \underbrace{1, \ldots, 1}_{a-1})
$$

where $\zeta\left(k_{1}, k_{2}, \ldots, k_{n}\right)$ is the multiple zeta value defined by

$$
\zeta\left(k_{1}, k_{2}, \ldots, k_{n}\right)=\sum_{m_{1}>m_{2}>\cdots>m_{n}>0} \frac{1}{m_{1}^{k_{1}} m_{2}^{k_{2}} \cdots m_{n}^{k_{n}}}
$$

for $k_{1} \geq 2$ and $k_{2}, \ldots, k_{n} \geq 1$.

(ii) (Aomoto [1, Drinfel'd [4])

$$
1-\sum_{k, l=1}^{\infty} \zeta(k+1, \underbrace{1, \ldots, 1}_{l-1}) X^{k} Y^{l}=\frac{\Gamma(1-X) \Gamma(1-Y)}{\Gamma(1-X-Y)} .
$$

A generalization of this formula can be found in [29].

Corollary 4.2 (Hoffman [8, Theorem 4.4]). Let $k, l$ be positive integers. Then

$$
l ! \zeta_{M T, k}(1, \ldots, 1 ; l)=k ! \zeta_{M T, l}(1, \ldots, 1 ; k) .
$$

This result is a special case of the duality of multiple zeta values (see [9, Corollary 6.2] or [36, p.510]).

Corollary 4.3. (i) Let $a, q$ be non-negative integers with $(a, q) \neq(0,0)$. Then

$$
I_{1, q}(2 a ; \mathbf{1})=(-1)^{q+a} \frac{q !(2 a) !}{(2 \pi)^{2 a}} \sum_{k=1}^{q} \frac{1}{k !} \sum_{\substack{q_{1}, \ldots, q_{k} \geq 1 \\ q_{1}+\cdots+q_{k}=q}} \sum_{\substack{a_{1}, \ldots, a_{k} \geq 0 \\ a_{1}+\cdots+a_{k}=a}} c_{q_{1}, a_{1}} \cdots c_{q_{k}, a_{k}},
$$

where $c_{1,0}=0, c_{m, 0}=\left(1-2^{1-m}\right) \zeta(m) / m$ for $m \geq 2$, and

$$
c_{m, n}=-\frac{1}{2^{m-1}}\left(\begin{array}{c}
m+2 n \\
m
\end{array}\right) \frac{\zeta(m+2 n)}{m+2 n}
$$

for $m \geq 1$ and $n \geq 1$. 
(ii) Let a, $r$ be positive integers. Then

$$
\begin{aligned}
\zeta_{M T, r}(1, \ldots, 1 ; a) \\
=r ! \sum_{k=1}^{\min \{r, a\}} \frac{(-1)^{k-1}}{k !} \sum_{\substack{r_{1}, \ldots, r_{k} \geq 1 \\
r_{1}+\cdots+r_{k}=r}} \sum_{\substack{a_{1}, \ldots, a_{k} \geq 1 \\
a_{1}+\cdots+a_{k}=a}} \prod_{j=1}^{k}\left(\begin{array}{c}
r_{j}+a_{j} \\
r_{j}
\end{array}\right) \frac{\zeta\left(r_{j}+a_{j}\right)}{r_{j}+a_{j}} .
\end{aligned}
$$

Proof. (i) Applying the Taylor expansion

$$
\log \Gamma(z+1)=-\gamma z+\sum_{k=2}^{\infty}(-1)^{k} \frac{\zeta(k)}{k} z^{k} \quad(|z|<1),
$$

we obtain

$$
\sum_{k=0}^{\infty} \sum_{l=0}^{\infty}(-1)^{k+l}(2 \pi)^{2 l} I_{1, k}(2 l ; \mathbf{1}) \frac{u^{k} v^{2 l}}{k !(2 l) !}=\exp \left(\sum_{m=1}^{\infty} \sum_{n=0}^{\infty} c_{m, n} u^{m} v^{2 n}\right)
$$

for $u, v \in \mathbb{C}$ with $|u|<1,|v|<1$ and $|u / 2 \pm v|<1$. Thus, we have the result.

(ii) The result follows in the same way as above.

Example 4.1. Some examples for the log sine integral will be stated in Propositions 4.8 and 4.13

Example 4.2. Let $r$ be a positive integer. Then:

(i) (Mordell [25, (5)]) $\zeta_{M T, r}(1, \ldots, 1 ; 1)=r ! \zeta(r+1)$.

(ii) (Subbarao-Sitaramachandrarao [31, (3.6)])

$$
\zeta_{M T, r}(1, \ldots, 1 ; 2)=\frac{r !}{2}\left\{(r+1) \zeta(r+2)-\sum_{k=2}^{r} \zeta(k) \zeta(r+2-k)\right\} .
$$

(iii) We see that

$$
\begin{aligned}
\zeta_{M T, r}(1, \ldots, 1 ; 3)=\frac{r !}{6}\{ & (r+1)(r+2) \zeta(r+3) \\
& -3 \sum_{\substack{k_{1}, k_{2} \geq 1 \\
k_{1}+k_{2}=r}}\left(k_{2}+1\right) \zeta\left(k_{1}+1\right) \zeta\left(k_{2}+2\right) \\
& \left.+\sum_{\substack{k_{1}, k_{2}, k_{3} \geq 1 \\
k_{1}+k_{2}+k_{3}=r}} \zeta\left(k_{1}+1\right) \zeta\left(k_{2}+1\right) \zeta\left(k_{3}+1\right)\right\} .
\end{aligned}
$$

By Corollary 4.2, this example is equivalent to Markett's result [20, Corollary 4.3] when $r \geq 2$.

4.2. Integrals $I_{p, 0}$. By (2.7), the integral $I_{p, 0}(\boldsymbol{a})$ can be expressed by the Riemann zeta values at positive even integers.

Example 4.3. Let $a, b, c, d$ be positive integers.

(i) $I_{1,0}(a)=0$ for all $a$.

(ii) If $a+b$ is even, then

$$
I_{2,0}(a, b)=(-1)^{\frac{a-b}{2}} \frac{2 a ! b !}{(2 \pi)^{a+b}} \zeta(a+b) .
$$


(iii) If $a+b+c$ is even, then

$$
\begin{aligned}
I_{3,0}(a, b, c)= & \frac{(-1)^{\frac{a+b-c+2}{2}} 4 a ! b ! c !}{(2 \pi)^{a+b+c}} \\
& \times \sum_{k}\left\{\left(\begin{array}{c}
a+b-1-2 k \\
a-1
\end{array}\right)+\left(\begin{array}{c}
a+b-1-2 k \\
b-1
\end{array}\right)\right\} \\
& \times \zeta(2 k) \zeta(a+b+c-2 k),
\end{aligned}
$$

where the sum is taken over all integers $k \in[0, \max \{a, b\} / 2]$.

(iv) If $a+b+c+d$ is even, then

$$
\begin{aligned}
I_{4,0}(a, b, c, d) & \\
= & \frac{(-1)^{\frac{a+b+c-d+2}{2}} 12 a ! b ! c ! d !}{(2 \pi)^{a+b+c+d}}\left[\left(\begin{array}{c}
a+b+c-3 \\
a-1, b-1, c-1
\end{array}\right) \zeta(2) \zeta(a+b+c+d-2)\right. \\
& -\frac{2}{3} \sum_{k, l}\left\{\left(\begin{array}{c}
a+b+c-1-2 k-2 l \\
a-1, b-2 k, c-2 l
\end{array}\right)+\left(\begin{array}{c}
a+b+c-1-2 k-2 l \\
a-2 l, b-1, c-2 k
\end{array}\right)\right. \\
& \left.\left.+\left(\begin{array}{c}
a+b+c-1-2 k-2 l \\
a-2 k, b-2 l, c-1
\end{array}\right)\right\} \zeta(2 k) \zeta(2 l) \zeta(a+b+c+d-2 k-2 l)\right],
\end{aligned}
$$

where the sum is taken over all non-negative integers $k, l$ such that $k+l<(a+b+$ c) $/ 2$.

4.3. Integrals $I_{p, 1}$. An explicit formula of the integral $I_{p, 1}$ is easily shown by Proposition 2.6.

Proposition 4.4. Let $p, b$ be positive integers and let $\boldsymbol{a}=\left(a_{1}, \ldots, a_{p}\right) \in\left(\mathbb{Z}_{\geq 1}\right)^{p}$. If $b$ is odd, then $I_{0,1}(b)=0$. Moreover, if $|\boldsymbol{a}|+b$ is odd, then

$$
I_{p, 1}(\boldsymbol{a} ; b)=\frac{(-1)^{\frac{|\boldsymbol{a}|-b+1}{2}} b !}{(2 \pi)^{|\boldsymbol{a}|+b-1}} \sum_{l}(-1)^{l}(|\boldsymbol{a}|-2 l) !(2 \pi)^{2 l} \mathcal{B}_{p}(\boldsymbol{a} ; l) \zeta(|\boldsymbol{a}|+b-2 l),
$$

where the sum is taken over all integers $l \in[0,|\boldsymbol{a}| / 2)$.

Proof. The result follows in the same way as in the proof of Proposition 2.5

Example 4.4. Let $a, b, c, d$ be positive integers.

(i) $I_{0,1}(a)=0$ for all $a$.

(ii) If $a+b$ is odd, then

$$
I_{1,1}(a ; b)=(-1)^{\frac{a-b+1}{2}} \frac{a ! b !}{(2 \pi)^{a+b-1}} \zeta(a+b) .
$$

(iii) If $a+b+c$ is odd, then

$$
\begin{aligned}
I_{2,1}(a, b ; c)= & \frac{(-1)^{\frac{a+b-c-1}{2}} 2 a ! b ! c !}{(2 \pi)^{a+b+c-1}} \\
& \times \sum_{k}\left\{\left(\begin{array}{c}
a+b-1-2 k \\
a-1
\end{array}\right)+\left(\begin{array}{c}
a+b-1-2 k \\
b-1
\end{array}\right)\right\} \\
& \times \zeta(2 k) \zeta(a+b+c-2 k),
\end{aligned}
$$

where the sum is taken over all integers in $[0, \max \{a, b\} / 2]$. 
(iv) If $a+b+c+d$ is odd, then

$$
\begin{aligned}
I_{3,1}(a, b, c ; d) & \\
= & \frac{(-1)^{\frac{a+b+c-d-1}{2}} 6 a ! b ! c ! d !}{(2 \pi)^{a+b+c+d-1}}\left[\left(\begin{array}{c}
a+b+c-3 \\
a-1, b-1, c-1
\end{array}\right) \zeta(2) \zeta(a+b+c+d-2)\right. \\
& -\frac{2}{3} \sum_{k, l}\left\{\left(\begin{array}{c}
a+b+c-1-2 k-2 l \\
a-1, b-2 k, c-2 l
\end{array}\right)+\left(\begin{array}{c}
a+b+c-1-2 k-2 l \\
a-2 l, b-1, c-2 k
\end{array}\right)\right. \\
& \left.\left.+\left(\begin{array}{c}
a+b+c-1-2 k-2 l \\
a-2 k, b-2 l, c-1
\end{array}\right)\right\} \zeta(2 k) \zeta(2 l) \zeta(a+b+c+d-2 k-2 l)\right],
\end{aligned}
$$

where the sum is taken over all non-negative integers $k, l$ such that $k+l<(a+b+$ c) $/ 2$.

4.4. $I_{p, 2}$ and $\zeta_{M T, 2}$. Unlike the previous subsections, it seems that the integral $I_{p, 2}$ is not always expressed by the Riemann zeta values. Most results for $I_{p, 2}$ mentioned below have equivalent results for $\zeta_{M T, 2}$ via the relation

$$
\zeta_{M T, 2}(a, b ; c)=\frac{(-1)^{\frac{a+b-c}{2}}(2 \pi)^{a+b+c-2}}{a ! b ! c !}\left(I_{1,2}(c ; a, b)-\pi^{2} I_{3,0}(a, b, c)\right)
$$

for positive integers $a, b, c$ satisfying that $a+b+c$ is even. Hence, the corresponding result for $\zeta_{M T, 2}$ is described together without proof.

Proposition 4.5. If $a+b$ is even, then

$$
I_{0,2}(a, b)=(-1)^{\frac{a-b}{2}} \frac{a ! b !}{2(2 \pi)^{a+b-2}} \zeta(a+b) .
$$

Proof. The result follows from $I_{0,2}(a, b)=\pi^{2} I_{2,0}(a, b)$ given by formula (2.2).

Proposition 4.6. (i) If $a$ is an even integer $\geq 2$, then

$$
I_{1,2}(a ; a, a)=\frac{(-1)^{\frac{a}{2}+1} 2(a !)^{3}}{3(2 \pi)^{3 a-2}} \sum_{k=0}^{a / 2}\left(\begin{array}{c}
2 a-1-2 k \\
a-1
\end{array}\right) \zeta(2 k) \zeta(3 a-2 k) .
$$

(ii) For an even integer $a \geq 2$,

$$
\zeta_{M T, 2}(a, a ; a)=\frac{4}{3} \sum_{k=0}^{a / 2}\left(\begin{array}{c}
2 a-1-2 k \\
a-1
\end{array}\right) \zeta(2 k) \zeta(3 a-2 k) .
$$

Proof. (i) Formula (2.3) shows that

$$
I_{1,2}\left(c_{1} ; c_{2}, c_{3}\right)+I_{1,2}\left(c_{2} ; c_{3}, c_{1}\right)+I_{1,2}\left(c_{3} ; c_{1}, c_{2}\right)=\pi^{2} I_{3,0}\left(c_{1}, c_{2}, c_{3}\right)
$$

for positive integers $c_{1}, c_{2}, c_{3}$. Hence, we obtain $I_{1,2}(a ; a, a)=\frac{\pi^{2}}{3} I_{3,0}(a, a, a)$, which yields the result.

Remark 4.2. Mordell proved the integral expression (3.9) of $\zeta_{M T, 2}(a, a ; a)$ for $a$ even, and he calculated only the case $a=2$ concretely. Carlitz [3] showed the explicit expression of the integral $I_{3,0}(a, b, c)$ for positive integers $a, b, c$. Although he did not describe an explicit formula of the zeta value, he quoted Mordell's work. (There is a misprint in the quotation [3, p.362].) After their studies, Subbarao and Sitaramachandrarao 31 . proved an explicit expression of the value $\zeta_{M T, 2}(a, b ; c)+$ $\zeta_{M T, 2}(b, c ; a)+\zeta_{M T, 2}(c, a ; b)$ for $a, b, c$ even, and specialized it to obtain a formula equivalent to (4.24). 
Proposition 4.7. Let $a$ be an even integer $\geq 2$.

(i) We have

$$
I_{1,2}(a+1 ; a, a-1)=\frac{(-1)^{\frac{a}{2}}(a+1) ! a !(a-1) !}{3(2 \pi)^{3 a-2}} \sum_{k=0}^{a / 2}\left(\begin{array}{c}
2 a-1-2 k \\
a-1
\end{array}\right) \zeta(2 k) \zeta(3 a-2 k) .
$$

(ii) (Huard-Williams-Zhang [10, p.116])

$$
\zeta_{M T, 2}(a-1, a ; a+1)=\frac{2}{3} \sum_{k=0}^{a / 2}\left(\begin{array}{c}
2 a-1-2 k \\
a-1
\end{array}\right) \zeta(2 k) \zeta(3 a-2 k) .
$$

Proof. (i) Applying Proposition 2.2, we have $I_{1,2}(a+1 ; a, a-1)=-\frac{a+1}{2 a} I_{1,2}(a ; a, a)$. Thus, the formula holds.

Proposition 4.8. Let $a$ be an even integer $\geq 2$.

(i) We obtain

$$
I_{1,2}(a ; 1,1)=\frac{(-1)^{\frac{a+2}{2}} a !}{(2 \pi)^{a}}\left(\frac{1}{2}(a+1) \zeta(a+2)-\sum_{\substack{k=3 \\ k: \text { odd }}}^{a-1} \zeta(k) \zeta(a+2-k)\right) .
$$

(ii) (Tornheim [32, Theorem 2])

$$
\zeta_{M T, 2}(1,1 ; a)=\frac{1}{2}(a-1) \zeta(a+2)-\sum_{\substack{k=3 \\ k: \text { odd }}}^{a-1} \zeta(k) \zeta(a+2-k) .
$$

Proof. (i) The result is an example of Corollary 4.3.

Proposition 4.9. Let $a$ and $b$ be positive integers. Suppose that $a+b$ is odd and $b>a$.

(i) We have

$$
I_{1,2}(1 ; a, b)=\frac{(-1)^{\frac{a-b-1}{2}} a ! b !}{2(2 \pi)^{a+b-1}}\left(\frac{1}{2}(b-a) \zeta(a+b+1)-\sum_{\substack{a<k \leq b \\ k: \text { odd }}} \zeta(k) \zeta(a+b+1-k)\right) .
$$

(ii) (Tornheim [32, Theorem 3])

$$
\begin{aligned}
\zeta_{M T, 2}(a, b ; 1)=(-1)^{a+1}( & \frac{1}{4}(a+b+2) \zeta(a+b+1)-\sum_{\substack{2 \leq k \leq a \\
k: \text { even }}} \zeta(k) \zeta(a+b+1-k) \\
& \left.-\frac{1}{2} \sum_{\substack{a<k \leq b \\
k: \text { odd }}} \zeta(k) \zeta(a+b+1-k)\right)
\end{aligned}
$$

Proof. (i) Proposition 2.2 shows that

$$
\begin{aligned}
I_{1,2}(1 ; c, d)= & -\frac{c}{d+1} I_{1,2}(1 ; c-1, d+1) \\
& -\frac{1}{d+1} I_{0,2}(c, d+1)+c \log \mathbf{S}_{c}(0) \log \mathbf{S}_{d+1}(0)
\end{aligned}
$$


for integers $c, d$ such that $c \geq 2, d \geq 1$ and $c+d$ is odd. Hence, by induction on $a$, we have

$$
\begin{aligned}
I_{1,2}(1 ; a, b)= & (-1)^{a-1} \frac{a ! b !}{(a+b-1) !} I_{1,2}(1 ; 1, a+b-1) \\
& +\sum_{k=1}^{a-1} \frac{(-1)^{k} a ! b !}{(a+1-k) !(b+k) !} I_{0,2}(a+1-k, b+k) \\
& +\sum_{k=1}^{a-1} \frac{(-1)^{k+1} a ! b !}{(a-k) !(b-1+k) !} \log \mathbf{S}_{a+1-k}(0) \log \mathbf{S}_{b+k}(0) .
\end{aligned}
$$

Thus, we obtain the result.

Remark 4.3. Tornheim gave expressions of $\zeta_{M T, 2}(1,1 ; a)$ and $\zeta_{M T, 2}(a, b ; 1)$ available for all positive integers $a, b$. In our cases, they coincide with the above formulas. This fact follows from the well-known formula

$$
\sum_{\substack{k=0 \\ k \text { :even }}}^{n} \zeta(k) \zeta(n-k)=\frac{1}{2}(n-1) \zeta(n)
$$

for an even integer $n \geq 4$.

Proposition 4.10. (i) $I_{1,2}(2 ; 3,1)=(2 \pi)^{-4}\left(\zeta(6)-6 \zeta(3)^{2}\right)$.

(ii) $\zeta_{M T, 2}(3,1 ; 2)=-\zeta(6) / 3+\zeta(3)^{2} / 2$.

Proof. (i) Proposition 2.2 shows

$$
I_{1,2}(2 ; 3,1)=-I_{1,2}(1 ; 2,3)-\frac{3}{2} I_{1,2}(2 ; 2,2) .
$$

Hence, we have the result.

Remark 4.4. Huard, Williams and Zhang gave an explicit evaluation of $\zeta_{M T, 2}(a, b ; c)$ into a linear combination of double zeta values $\zeta_{M T, 2}(k, 0 ; l)=\zeta(l, k)$ (see [10, (1.6)]). For example, $\zeta_{M T, 2}(3,1 ; 2)=2 \zeta(5,1)+\zeta(4,2)+\zeta(3,3)$. Moreover, they showed that $\zeta_{M T, 2}(a, b ; c)$ can be expressed by the Riemann zeta values when $a+$ $b+c \leq 7$. Hence, Proposition 4.10 (ii) is a known fact.

4.5. $I_{p, 3}$ and $\zeta_{M T, 3}$. We study the expression of $I_{p, 3}$ by the Riemann zeta values in the same way as in the previous subsection. In order to connect it to the result for $\zeta_{M T, 3}$, we apply the formula

$$
\begin{aligned}
\zeta_{M T, 3}(a, b, c ; d) \\
=\frac{(-1)^{\frac{a+b+c-d+1}{2}}(2 \pi)^{a+b+c+d-3}}{a ! b ! c ! d !} \\
\quad \times\left\{I_{1,3}(d ; a, b, c)-\pi^{2}\left(I_{3,1}(a, b, d ; c)+I_{3,1}(b, c, d ; a)+I_{3,1}(c, a, d ; b)\right)\right\}
\end{aligned}
$$

for positive integers $a, b, c, d$ satisfying that $a+b+c+d$ is odd.

Proposition 4.11. If $a+b+c$ is odd, then

$$
I_{0,3}(a, b, c)=\pi^{2}\left(I_{2,1}(a, b ; c)+I_{2,1}(b, c ; a)+I_{2,1}(c, a ; b)\right),
$$

where the integrals on the right hand side are expressed by the Riemann zeta values such as (4.23). 
Proof. The result follows from formula (2.2).

Proposition 4.12. (i) If $a$ is an odd integer $\geq 3$, then

$$
\begin{aligned}
& I_{1,3}(1 ; a-1, a, a) \\
& \quad=(-1)^{\frac{a+1}{2}} \frac{(a-1) !(a !)^{2}}{(2 \pi)^{3 a-3}}\left\{\frac{1}{3} \zeta(a)^{3}+\sum_{k=0}^{(a-1) / 2}\left(\begin{array}{c}
2 a-1-2 k \\
a-1
\end{array}\right) \zeta(2 k) \zeta(3 a-2 k)\right\} .
\end{aligned}
$$

(ii) For an odd integer $a \geq 3$,

$$
\zeta_{M T, 3}(a-1, a, a ; 1)=\frac{1}{3} \zeta(a)^{3} .
$$

Proof. (i) Proposition 2.2 yields that

$$
I_{1,3}(1 ; a-1, a, a)=-\frac{1}{3 a} I_{0,3}(a, a, a)+\frac{1}{3} a^{2} \log ^{3} \mathbf{S}_{a}(0) .
$$

Thus, we have the result.

Remark 4.5. The result for $\zeta_{M T, 3}$ can be shown by a general argument in the next subsection.

Proposition 4.13. (i) If $a$ is an even integer $\geq 2$, then

$$
\begin{aligned}
I_{1,3}(a ; 1,1,1)=\frac{(-1)^{\frac{a}{2}} a !}{(2 \pi)^{a}}\{ & \frac{3}{2} \zeta(2) \zeta(a+1)+\frac{1}{4}(a+1)(a+2) \zeta(a+3) \\
& -\frac{3}{2} \sum_{\substack{k_{1}, k_{2} \geq 1 \\
k_{1}+k_{2}=a / 2}}\left(2 k_{1}+1\right) \zeta\left(2 k_{1}+2\right) \zeta\left(2 k_{2}+1\right) \\
& \left.+\sum_{\substack{k_{1}, k_{2}, k_{3} \geq 1 \\
k_{1}+k_{2}+k_{3}=a / 2}} \zeta\left(2 k_{1}+1\right) \zeta\left(2 k_{2}+1\right) \zeta\left(2 k_{3}+1\right)\right\} .
\end{aligned}
$$

(ii) (Markett [20, Corollary 4.3]) For an even integer $a \geq 2$,

$$
\begin{aligned}
\zeta_{M T, 3}(1,1,1 ; a)= & -3 a \zeta(2) \zeta(a+1)+(a+1)(a+2) \zeta(a+3) \\
& -\frac{3}{2} \sum_{\substack{k_{1}, k_{2} \geq 1 \\
k_{1}+k_{2}=a / 2}}\left(a+2 k_{2}-1\right) \zeta\left(2 k_{1}+2\right) \zeta\left(2 k_{2}+1\right) \\
& +\sum_{\substack{k_{1}, k_{2}, k_{3} \geq 1 \\
k_{1}+k_{2}+k_{3}=a / 2}} \zeta\left(2 k_{1}+1\right) \zeta\left(2 k_{2}+1\right) \zeta\left(2 k_{3}+1\right) .
\end{aligned}
$$

Proof. (i) The result is an example of Corollary 4.3.

Remark 4.6. Markett proved an expression of $\zeta_{M T, 3}(1,1,1 ; a)$ for all positive integers $a$, which is equivalent to the above formula when $a$ is even. 
Theorem 5. (i) If $a$ is an even integer $\geq 2$, then

$$
\begin{aligned}
I_{1,3}(1 ; 1,1, a)=\frac{(-1)^{\frac{a}{2}} a !}{(2 \pi)^{a}}\{ & \frac{2 a-1}{2} \zeta(2) \zeta(a+1)-\frac{2 a^{2}+6 a+1}{6} \zeta(a+3) \\
& +\frac{1}{2} \sum_{\substack{k_{1}, k_{2} \geq 1 \\
k_{1}+k_{2}=a / 2}}\left(a+2 k_{2}-1\right) \zeta\left(2 k_{1}+2\right) \zeta\left(2 k_{2}+1\right) \\
& \left.-\frac{1}{3} \sum_{\begin{array}{c}
k_{1}, k_{2}, k_{3} \geq 1 \\
k_{1}+k_{2}+k_{3}=a / 2
\end{array}} \zeta\left(2 k_{1}+1\right) \zeta\left(2 k_{2}+1\right) \zeta\left(2 k_{3}+1\right)\right\} .
\end{aligned}
$$

(ii) For an even integer $a \geq 2$,

$$
\begin{aligned}
\zeta_{M T, 3}(a, 1,1 ; 1)= & a \zeta(2) \zeta(a+1)-\frac{1}{6}(a-1)(a+4) \zeta(a+3) \\
& -\frac{1}{2} \sum_{\substack{k_{1}, k_{2} \geq 1 \\
k_{1}+k_{2}=a / 2}}\left(a-6 k_{2}+3\right) \zeta\left(2 k_{1}+2\right) \zeta\left(2 k_{2}+1\right) \\
& +\frac{1}{3} \sum_{\substack{k_{1}, k_{2}, k_{3} \geq 1 \\
k_{1}+k_{2}+k_{3}=a / 2}} \zeta\left(2 k_{1}+1\right) \zeta\left(2 k_{2}+1\right) \zeta\left(2 k_{3}+1\right) .
\end{aligned}
$$

Proof. (i) Formula (2.3) shows that

$$
I_{1,3}(1 ; 1,1, a)=\pi^{2} I_{3,1}(a, 1,1 ; 1)+\frac{\pi^{2}}{3} I_{3,1}(1,1,1 ; a)-\frac{1}{3} I_{1,3}(a ; 1,1,1) .
$$

By simple calculation, we obtain the result.

Concerning the zeta value $\zeta_{M T, 3}(a, 1,1 ; 1)$ with $a$ odd, we can show that

$$
\begin{aligned}
\zeta_{M T, 3}(1,1,1 ; 1) & =6 \zeta(4), \\
\zeta_{M T, 3}(3,1,1 ; 1) & =\frac{89}{24} \zeta(6)-\zeta(3)^{2} .
\end{aligned}
$$

However, in the case where $a$ is an odd integer $\geq 5$, it does not appear that $\zeta_{M T, 3}(a, 1,1 ; 1)$ can be expressed by the Riemann zeta values. These can be shown by the next result.

Proposition 4.14. If $a$ is an odd integer $\geq 1$, then

$$
\begin{aligned}
\zeta_{M T, 3}(a, 1,1 ; 1)= & 2 \zeta_{M T, 2}(a, 1 ; 2) \\
& +\frac{5}{2} \zeta(2) \zeta(a+1)+\frac{1}{8}(a-3)(a+4) \zeta(a+3) \\
& -\frac{1}{2}(a+1) \sum_{\substack{k_{1}, k_{2} \geq 1 \\
k_{1}+k_{2}=(a+1) / 2}} \zeta\left(2 k_{1}+1\right) \zeta\left(2 k_{2}+1\right) \\
& +\sum_{\substack{k_{1}, k_{2}, k_{3} \geq 1 \\
k_{1}+k_{2}+k_{3}=(a+1) / 2}} \zeta\left(2 k_{1}\right) \zeta\left(2 k_{2}+1\right) \zeta\left(2 k_{3}+1\right) .
\end{aligned}
$$


Proof. The result follows from equation (3.17). Here, we may apply the formula

$$
\sum_{\substack{k=2 \\ k: \text { :even }}}^{n} k \zeta(k) \zeta(n-k)=\frac{1}{4} n(n-1) \zeta(n)
$$

for an even integer $n \geq 4$.

4.6. Other cases. Here we display some results. We see that $\zeta_{M T, r}\left(c_{1}, \ldots, c_{r} ; c_{r+1}\right)$ can be expressed by the Riemann zeta values if $c_{1}, \ldots, c_{r+1} \in \mathbb{Z}_{\geq 1}$ and $\sum_{j=1}^{r+1} c_{j} \leq 6$, by combining some of the results mentioned above and by the following.

Proposition 4.15. $\zeta_{M T, 4}(2,1,1,1 ; 1)=\zeta(6) / 2+6 \zeta(3)^{2}$.

Proof. Applying Theorem 4, we can show that

$$
\begin{aligned}
\zeta_{M T, 4}(2,1,1,1 ; 1)= & \frac{1}{4} \zeta_{M T, 4}(1,1,1,1 ; 2)-9 \zeta_{M T, 2}(1,1 ; 4) \\
& +\frac{5 \pi^{2}}{4} \zeta_{M T, 2}(1,1 ; 2)+3 \zeta_{M T, 2}(1,2 ; 3) \\
& -\frac{\pi^{2}}{2} \zeta_{M T, 2}(2,1 ; 1)+8 \pi^{6} I_{5,0}(1,1,1,1,2) .
\end{aligned}
$$

Thus, we have the result.

Remark 4.7. As the referee pointed out, any Mordell-Tornheim zeta value with positive integer arguments can be written as a linear combination (with integer coefficients) of multiple zeta values (4.21). The referee also proved Proposition 4.15 by expanding into multiple zeta values and using known results about them, as follows. By (4.25) below, we obtain

$$
\zeta_{M T, 4}(2,1,1,1 ; 1)=\zeta_{M T, 4}(1,1,1,1 ; 2)+3 \zeta_{M T, 4}(2,1,1,0 ; 2) .
$$

The first term on the right hand side is $24 \zeta(5,1)$ by (4.20), and the second term is

$$
\begin{aligned}
& \sum_{n_{1}, n_{2}, n_{3}, n_{4} \geq 1} \frac{3}{n_{1}^{2} n_{2} n_{3} s_{4}^{2}}=\sum_{n_{1}, n_{2}, n_{3}, n_{4} \geq 1} \frac{3}{n_{1} n_{2} n_{3} s_{3} s_{4}^{2}}+\sum_{n_{1}, n_{2}, n_{3}, n_{4} \geq 1} \frac{6}{n_{1} n_{2} s_{3}^{2} s_{4}^{2}}+\sum_{n_{1}, n_{2}, n_{3}, n_{4} \geq 1} \frac{6}{n_{1}^{2} n_{2} s_{3} s_{4}^{2}} \\
= & \sum_{n_{1}, n_{2}, n_{3}, n_{4} \geq 1} \frac{18}{n_{1}^{2} s_{2} s_{3} s_{4}^{2}}+\sum_{n_{1}, n_{2}, n_{3}, n_{4} \geq 1} \frac{6}{n_{1} s_{2} s_{3}^{2} s_{2}^{2} s_{2} s_{3} s_{4}^{2}}+6 \zeta(2,1,1,2)+\sum_{n_{1}, n_{2}, n_{3}, n_{4} \geq 1} \frac{12}{n_{1} s_{2}^{2} s_{3} s_{4}^{2}} \\
= & 18 \zeta(2,2,1,1)+6 \zeta(2,1,1,2)+12 \zeta(2,1,2,1) \\
= & 18 \zeta(4,2)+6 \zeta(2,4)+12 \zeta(3,3),
\end{aligned}
$$

where we have written $s_{i}$ for $n_{1}+n_{2}+\cdots+n_{i}$, and in the last step used the duality of multiple zeta values. Hence

$$
\zeta_{M T, 4}(2,1,1,1 ; 1)=24 \zeta(5,1)+18 \zeta(4,2)+12 \zeta(3,3)+6 \zeta(2,4) .
$$

Using well-known relations among double zeta values, the right hand side coincides with $\zeta(6) / 2+6 \zeta(3)^{2}$.

Proposition 4.12 (ii) is generalized as follows. 
Proposition 4.16. Let $r, a$ be integers $\geq 2$. Then

$$
\zeta_{M T, r}(a-1, a, \ldots, a ; 1)=\frac{1}{r} \zeta(a)^{r} .
$$

Proof. Note that

$$
\sum_{j=1}^{r} \zeta_{M T, r}\left(c_{1}, \ldots, c_{j-1}, c_{j}-1, c_{j+1}, \ldots, c_{r} ; c_{r+1}\right)=\zeta_{M T, r}\left(c_{1}, \ldots, c_{r} ; c_{r+1}-1\right) .
$$

Taking $c_{1}=\cdots=c_{r}=a$ and $c_{r+1}=1$, we obtain the formula.

Remark 4.8. Proposition 4.16 can be proved via the properties of the integral $I_{p, q}$ : Theorem 1 and Propositions 2.2 and 2.3. However, such a proof is more complicated than the one above. Hence, it is omitted here.

\section{ACKNOWLEDGEMENTS}

The author expresses gratitude to Dr. Takashi Nakamura and Professor Hirofumi Tsumura for their valuable comments, and to the referee for useful suggestions.

\section{REFERENCES}

[1] K. Aomoto, Special values of hyperlogarithms and linear difference schemes, Illinois J. Math. 34 (1990), 191-216. MR 1046562 (91f:33012)

[2] T. M. Apostol, Introduction to analytic number theory, Springer, New York, 1976. MR0434929 (55:7892)

[3] L. Carlitz, Note on the integral of the product of several Bernoulli polynomials, J. London Math. Soc. 34 (1959), 361-363. MR0107022 (21:5750)

[4] V. G. Drinfel'd, On quasitriangular quasi-Hopf algebras and a group closely connected with $\operatorname{Gal}(\overline{\mathbf{Q}} / \mathbf{Q})$, Leningrad Math. J. 2 (1991), 829-860. MR.1080203 (92f:16047)

[5] O. Espinosa and V. H. Moll, The evaluation of Tornheim double sums, part 1, J. Number Theory 116 (2006), 200-229. MR2197867 (2007c:11100)

[6] L. Euler, De summis serierum numeros Bernoullianos involventium, Novi Comm. Acad. Sci. Petropolitanae 14 (1769), 129-167 (Opera Omnia I-15, pp. 91-130).

[7] L. Euler, Exercitationes analyticae, Novi Comm. Acad. Sci. Petropolitanae 17 (1772), 173204 (Opera Omnia I-15, pp.131-167).

[8] M. E. Hoffman, Multiple harmonic series, Pacific J. Math. 152 (1992), 275-290. MR1141796 (92i:11089)

[9] M. E. Hoffman, The algebra of multiple harmonic series, J. Algebra 194 (1997), 477-495. MR1467164 (99e:11119)

[10] J. G. Huard, K. S. Williams and N.-Y. Zhang, On Tornheim's double series, Acta Arith. 75 (1996), 105-117. MR.1379394 (97f:11073)

[11] Y. Komori, An integral representation of the Mordell-Tornheim double zeta function and its values at non-positive integers, Ramanujan J. 17 (2008), 163-183. MR2452649(2009h:11147)

[12] Y. Komori, K. Matsumoto and H. Tsumura, Zeta-functions of root systems, in: Proceedings of the Conference on $L$-functions, Fukuoka, 2006 (eds. L. Weng and M. Kaneko), World Scientific, 2007, 115-140. MR2310292(2008h:11094)

[13] S. Koyama and N. Kurokawa, Euler's integrals and multiple sine functions, Proc. Amer. Math. Soc. 133 (2004), 1257-1265.

[14] N. Kurokawa, K. Mimachi and M. Wakayama, Jackson's integral of the Hurwitz zeta function, Rend. Circ. Mat. Palermo (2) 56 (2007), 43-56. MR2313770 (2008e:11104)

[15] N. Kurokawa and H. Ochiai, Generalized Kinkelin's formulas, Kodai Math. J. 30 (2007), 195-212. MR2343417 (2008i:33004)

[16] N. Kurokawa, H. Ochiai and M. Wakayama, Milnor's multiple gamma functions, J. Ramanujan Math. Soc. 21 (2006), 153-167. MR 2244542(2007f:11101)

[17] N. Kurokawa and M. Wakayama, A $q$-logarithmic analogue of Euler's sine integral, Rend. Sem. Mat. Univ. Padova 114 (2005), 51-62. MR2207861 (2007d:33004) 
[18] N. Kurokawa and M. Wakayama, Period deformations and Raabe's formulas for generalized gamma and sine functions, Kyushu J. Math. 62 (2008), 171-187. MR2413789 (2009c:11134)

[19] L. Lewin, Polylogarithms and associated functions, Elsevier, North Holland, New York, 1981. MR618278 (83b:33019)

[20] C. Markett, Triple sums and the Riemann zeta function, J. Number Theory 48 (1994), 113132. MR1285535(95f:11067)

[21] K. Matsumoto, On the analytic continuation of various multiple zeta-functions, in: Number Theory for the Millennium II, Proc. Millennial Conference on Number Theory, UrbanaChampaign, 2000 (eds. M. A. Bennett et al.), A. K. Peters, 2002, 417-440. MR 1956262 (2004a:11094)

[22] K. Matsumoto, On Mordell-Tornheim and other multiple zeta-functions, in: Proceedings of the Session in Analytic Number Theory and Diophantine Equations (eds. D. R. HeathBrown and B. Z. Moroz), Bonner Math. Schriften 360, Bonn, 2003, no. 25, 17pp. MR2075634 (2005f:11198)

[23] K. Matsumoto, T. Nakamura, H. Ochiai and H. Tsumura, On value-relations, functional relations and singularities of Mordell-Tornheim and related triple zeta-functions, Acta Arith. 132 (2008), 99-125. MR2395147 (2009c:11139)

[24] J. Milnor, On polylogarithms, Hurwitz zeta functions, and the Kubert identities, Ens. Math. 29 (1983), 281-322. MR719313 (86d:11007)

[25] L. J. Mordell, On the evaluation of some multiple series, J. London Math. Soc. 33 (1958), 368-371. MR0100181 (20:6615)

[26] L. J. Mordell, Integral formulae of arithmetical character, J. London Math. Soc. 33 (1958), 371-375. MR0100052 (20:6488)

[27] T. Nakamura, A functional relation for the Tornheim double zeta function, Acta Arith. 125 (2006), 257-263. MR2276193(2007h:11111)

[28] N. E. Nörlund, Vorlesungen über Differenzenrechnung, Springer, Berlin, 1924.

[29] Y. Ohno and D. Zagier, Multiple zeta values of fixed weight, depth, and height, Indag. Math. (N.S.) 12 (2001), 483-487. MR1908876 (2003e:11094)

[30] A. Petojević and H. M. Srivastava, Computation of the Mordell-Tornheim zeta values, Proc. Amer. Math. Soc. 136 (2008), 2719-2728. MR2399033(2009a:11191)

[31] M. V. Subbarao and R. Sitaramachandrarao, On some infinite series of L. J. Mordell and their analogues, Pacific J. Math. 119 (1985), 245-255. MR797027 (87c:11091)

[32] L. Tornheim, Harmonic double series, Amer. J. Math. 72 (1950), 303-314. MR0034860 $(11: 654 a)$

[33] H. Tsumura, On some combinatorial relations for Tornheim's double series, Acta Arith. 105 (2002), 239-252. MR1931792 (2003i:11134)

[34] H. Tsumura, On Mordell-Tornheim zeta values, Proc. Amer. Math. Soc. 133 (2005), 23872393. MR2138881 (2006k:11179)

[35] H. Tsumura, On functional relations between the Mordell-Tornheim double zeta functions and the Riemann zeta function, Math. Proc. Cambridge Philos. Soc. 142 (2007), 395-405. MR2329691(2008d:11103)

[36] D. Zagier, Values of zeta functions and their applications, in "First European Congress of Mathematics", Vol. II, pp. 497-512, Birkhauser Boston, Cambridge, MA, 1994. MR.1341859 (96k:11110)

Department of Mathematics, Tokyo Institute of Technology, O-okayama, MeguroKU, TOKYO 152-8551, JAPAN

E-mail address: onodera@math.titech.ac.jp 